\title{
Observações preliminares sôbre caponização de trangos "Leghorn" com estrogênios
}

\author{
E. A. GRANER e A. P. TRIVELIN \\ Escola Superior de Agricultura "Luiz de Queiroz" \\ Universidade de São Paulo
}

\section{INDICE}
1) Introdução $\ldots \ldots \ldots \ldots \ldots \ldots \ldots \ldots \ldots \ldots \ldots, 30$
2) Material e Método $\ldots \ldots \ldots \ldots \ldots \ldots \ldots \ldots \ldots, 30$
3) Resultados $\ldots \ldots \ldots \ldots \ldots \ldots \ldots \ldots \ldots \ldots \ldots \ldots, 31$

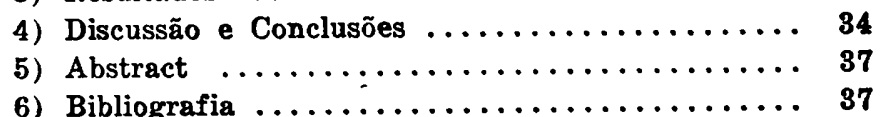

Trabalho da Secção de Avicultura 


\section{1 - INTRODUÇAOO}

A castração de frangos constitui hoje uma prática bastante conhecida e generalizada. Ela só não vem sendo empregada, no nosso meio, em maior escala, devido principalmente à falta de mercado apropriado para os produtos castrados e a certa dificuldade na técnica da castração, que exige algum treinamento do operador, se bem que a operação seja relativamente simples. Daí o interêsse na pesquisa de outros meios que, produzindo pràticamente os mesmos resultados, simplifiquem o trabalho da operação de castrar. Nestes últimos anos, atenção especial vem sendo dispensada ao emprêgọ de hormônios femininos (estrogênios) para substituir a castração cirúrgica. $O$ efeito dos estrogênios é o de paralizar o desenvolvimento dos órgãos reprodutores masculinos (testículos), não sendo porisso uma verdadeira castração, se bem que o método venha sendo generalizado com o nome de castração química ou fisiológica. A designação "ceva de aves" foi também empregada. Neste artigo preferimos chamá-lo de caponização por meio de estrogênios. A expressão "caponização" é de uso corrente em avicultura e significa produção de capões. Evidentemente não importa porque meio o "capão" tenha sido produzido.

Grande é o número de trabalhos publicados sôbre o assunto, principalmente na literatura norte-americana. Não é nossa intenção fazer, neste pequeno artigo, uma revisão completa dessa bibliografia, mas sim comparar os nossos resultados com aquêles já obtidos por outros autôres. Nossas observações baseiam-se no emprêgo de um preparado sintético, lançado recentemente no mercado brasileiro e comercialmente designado por "Capastrol", o qual foi aplicado em frangos "Leghorn" disponíveis para a realização das experiências preliminares.

\section{2 - MATERIAL E MÉTODO}

Frangos da raça "Leghorn", de aproximadamente 3 meses de idade, num total de 40 , foram separados em dois lotes de 20 cada um, de maneira que cada lote abrangesse, mais ou menos, a mesma amplitude de variação, com relação ao tamanho das aves. Esses frangos apresentavam a crista razoavelmente desenvolvida, num tamanho que variava em tôrno de 50 milímetros, no maior comprimento, por 25 milímetros 
na maior altura. Um dos lotes permaneceu como testemunha e ao outro foram aplicados comprimidos de "Capastrol", produto sintético recentemente lançado no mercado pela Companhia Química Rhodia Brasileira, de São Paulo. Os comprimidos de "Capastrol", de cerca de $5 \mathrm{~m} / \mathrm{m}$ de diâmetro por $2 \mathrm{~m} / \mathrm{m}$ de espessura, encerram, em sua composição, como elemento básico, o dietilestilbestrol ou mais simplesmente estilbestrol, derivado dietílico do paradiidroxiestilbesteno. Cada frango tratado recebeu um comprimido, o qual foi introduzido no pescoço, debaixo da pele. A técnica empregada para a colocação dos comprimidos foi bastante simples. Com uma tesoura de ponta redonda, foi feita na pele um pequeno corte, suficiente para dar passagem ao comprimido, e com uma das fôlhas da mesma tesoura, foi o comprimido empurrado para baixo, numa distância de aproximadamente 2 a 3 centímetros, sem necessidade de ulterior sutura da fenda feita. Outras técnicas para a introdução do comprimido têm sido aconselhadas, como por exemplo aquela descrita por WAITSMAN (22), por meio do "pellet injector", aparelho fabricado nos Estados Unidos, com o qual o comprimido é empurrado com uma agulha manejada por um pequeno êmbolo. Os frangos, numerados individualmente, tanto os tratados como os não tratados, permaneceram em gaiolas, com separação entre os lotes, por cêrca de 6 semanas, tempo que durou a experiência. O pêso individual foi tomado semanalmente e o tamanho da crista foi medido quinzenalmente.

\section{3 - RESULTADOS}

As observações realizadas centralizaram-se no efeito que os comprimidos poderiam produzir no tamanho da crista, no ganho em pêso e no tamanho dos testículos. Elas serão relatadas separadamente, para maior facilidade de explanação.

\section{a) Tamanho da crista}

O tamanho da crista (dentro da mesma raça) é um teste hormonal bem característico e conhecido.

No início da experiência, tomámos a medida das cristas de todos os frangos, componentes dos dois lotes em observação. As medidas foram feitas em milímetros, tirando-se sempre, para cada frango, o maior comprimento e a maior altura. Das 20 cristas medidas em cada lote, fizemos as médias dêsses dois valores, as quais estão incluidas na figura 1. Como podemos ver, 
- tamanho da crista, quanto ao seu maior comprimento e sua maior altura, foi pràticamente o mesmo, para os dois lotes considerados. O maior comprimento oscilou em torno de 53,4 e 52,3 milímetros e a maior altura em volta de 25,9 e $23,4 \mathrm{mi}$ límetros. No final da primeira semana de tratamento, notamos, em todos os frangos tratados, uma descoloração acentuada das cristas, bem como o seu murchamento progressivo a partir da parte dentada. Duas semanas após o tratamento, as cristas foram novamente medidas e os resultados, sob a forma de médias, estão reunidos também na figura 1. Pudemos constatar que, 15 dias após o recebimento dos comprimidos, o tamanho da crista do lote tratado apresentou uma diferença bastante grande daquele apresentado pelo lote testemunha. Assim é que o ganho, em comprimento e altura, após 15 dias, foi de 19,3 e 11,8 milímetros respectivamente, para o lote não tratado. No lote tratado, não houve aumento do tamanho das cristấs, mas sim uma pequena redução de tamanho, constatada nas duás medidas. A diferença, após 15 dias de tratamento, é bastante grande, dispensando uma análise estatística. $O$ valor médio, para o lote tratado, foi tomado de 19 frangos, tendo um dêles sido excluido, por apresentar a crista sem murchamento, bastante colorida e de um tamanho que muito se aproximava àquele do lote não tratado. Tratava-se, muito provavelmente, de um frango onde o comprimido deixou de atuar, seja por perda, que não pode ser excluida, pois é possível que o comprimido tenha saido pelo orifício de entrada ou por falta de absorção pela pele.

Após 30 dias de tratamento, a crista, nos 2 lotes, foi novamente medida e os resultados estão reunidos à direita da figura 1. Verificámos que, no lote não tratado, a crista continuou a se desenvolver, alcançando um comprimento médio de 80,1 milímetros e uma altura de 44,5 milímetros. O tamanho da crista, proporcionalmente aos valores encontrados, está representado na mesma figura 1 . A crista, no lote tratado, mostrouse paralisada quanto ao seu crescimento, tendo havido mesmo uma redução de tamanho, caracterizada pela diferença de 10 milímetros quanto ao comprimento e 4 milímetros quanto à altura, em comparaçã̃o ao tamanho inicial. A média do lote tratado foi tomada de 15 frangos, 5 dêles sendo eliminados dêsse cômputo por apresentarem, nessa época, as cristas bastante vermelhas e grandes. Esses frangos indicam, aos 30 dias de tratamento, provável reação individual, pois que êles se mostraram afetados pelos comprimidos, nos primeiros 15 dias. $\mathrm{Na}$ experiência realizada não foi feita nova aplicação de compri- 
mido mas, do ponto de vista econômico, poder-se-á fazê-la, depois dos primeiros 30 dias, nos frangos que apresentarem crista muito desenvolvida.

\section{b) Ganho em pêso}

A análise do ganho em pêso, semanal, ficou prejudicada na presente experiência, devido ao fato dos frangos ficarem atacados, na bateria, por "bouba epiteliomatosa". Ambos os lotes mostraram-se igualmente atacados, relativamente ao número de frangos doentes. $O$ lote que recebeu os comprimidos mostrou-se mais sensível, no que se refere ao aumento de pêso das aves. Apesar disso, uma análise dos resultados, nos frangos que não se mostraram muito prejudicados pela doença (9 frangos no lote tratado e 16 frangos no lote controle, foi feita. O ganho médio semanal, para os 2 lotes, acha-se representado no quadro n. 1. Pcdemos constatar por êsse quadro, que o ganho médio, após a 1a. semana de tratamento, foi razoavelmente o mesmo para os 2 lotes. Já na 2a. semana após o tratamento, êsse valor para o lote tratado, foi sensivelmente menor que aquêle do lote não tratado, indicando que os frangos que receberam os comprimidos passaram por um período de forte reação ao tratamento. Nas semanas seguintes, o ganho em pêso foi práticamente o mesmo para ambos os lotes. Parece assim que o período de maior reação dos frangos tratados foi aquêle compreendido pela segunda semana de tratamento.

Uma análise da variância dos 25 frangos analisados (tratados e não tratados), cujos resultados estão reunidos no quadro n. 2, mostra que não houve diferença esiatística significativa entre os 25 frangos e entre os 2 lotes. Assim, no final da experiência, o ganho médio de cada frango foi o mesmo nos 2 lotes. Verifica-se então que, considerando apenas o aumento em pêso, tanto os frangos que receberam comprimidos, como os frangos não tratados, não apresentaram diferença significativa. Entretanto, observações bastante gerais pareceram indicar uma ossatura mais enfraquecida nos frangos tratados e, consequentemente, um relativo aumento do vestimento de carne. Também, essa carne mostrou-se à primeira vista, mais saborosa, devido, naturalmente, a um maior acúmulo de gordura.

Analisando os 2 lotes separadamente, pudemos constatar, por meio da análise da variância que, no lote contrôle (quadro n. 3), a diferença entre frangos continuou estatisticamente insignificante. Já a análise entre as semanas mostrou o valor de $F$ estatisticamente significativo, indicando que, para ês- 
QUADRO N. 1

GANHO MÉDIO POR SEMANA, EM GRAMAS

\begin{tabular}{l|r|r|r|r|r}
\hline \multirow{2}{*}{ Lotes } & \multicolumn{5}{|c}{ Sem 3 nas } \\
\cline { 2 - 5 } & 1a. & 2a. & 3a. & $4 a$. & $5 a$. \\
\hline Testemunha & 116,87 & 109,37 & 73,12 & 95,62 & 78,75 \\
Tratado & 131,10 & 52,22 & 78,88 & 60,00 & 71,11 \\
\hline
\end{tabular}

QUADRO N. 2

\begin{tabular}{|c|c|c|c|c|c|}
\hline 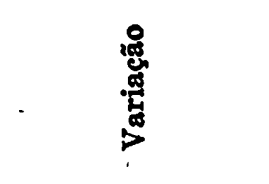 & 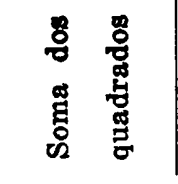 & 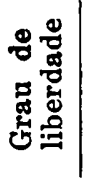 & 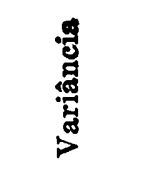 & 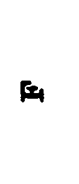 & 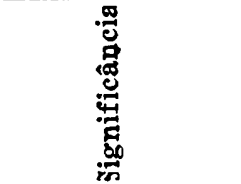 \\
\hline Total & $284.764,80$ & 109 & $2.612,52$ & - & \\
\hline Entre Frangos & $71.644,80$ & 24 & $2.985,20$ & 1,22 & Insignificante \\
\hline Entre Semanas & $7.449,80$ & 1 & $7.449,80$ & 3,04 & Insignificante \\
\hline Êrro & $205.670,20$ & 84 & $2.448,45$ & - & 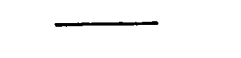 \\
\hline
\end{tabular}


QUADRO N. 3

\begin{tabular}{|c|c|c|c|c|c|}
\hline 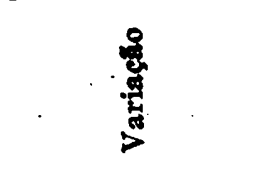 & 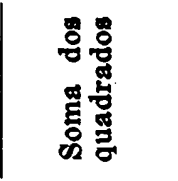 & 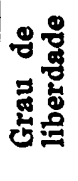 & 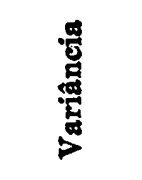 & E & 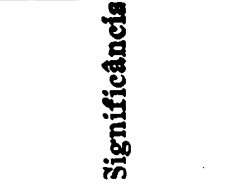 \\
\hline Total & $167.595,00$ & 79 & $2.121,45$ & - & \\
\hline Entre Frangos & $46.315,00$ & 15 & $3.087,66$ & 1,88 & Insignificante \\
\hline Entre Semanas & $22.845,00$ & 4 & $5.711,25$ & 3,48 & Significante \\
\hline Erro & $98.435,00$ & 60 & $1.640,58$ & - & \\
\hline
\end{tabular}

QUADRO N. 4

\begin{tabular}{|c|c|c|c|c|c|}
\hline 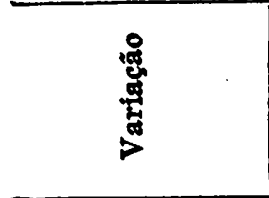 & 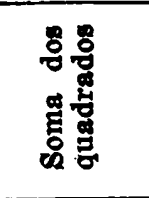 & 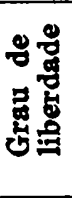 & 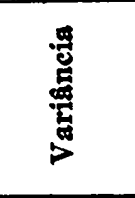 & m & 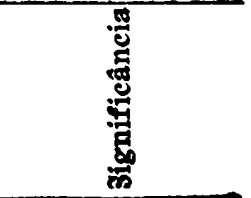 \\
\hline Total & $62.888,89$ & 35 & $1.796,81$ & - & \\
\hline Entre Frangos & $41.338,89$ & 8 & $5.167,36$ & 6,97 & Significante \\
\hline Entre Semanas & $3.755,55$ & 3 & $1.251,85$ & 1,68 & Insignificante \\
\hline Erro & $17.794,45$ & 24 & 741,43 & - & \\
\hline
\end{tabular}


te lote, o ganho médio semanal foi diferente, nas diferentes semanas de tratamento.

No que se refere ao lote tratado, uma inspeção do quadro n. 1 mostra que o ganho médio da 1a. semana de tratamento foi sensivelmente melhor que aquêle das demais semanas. Uma análise da variância (quadro n. 4) mostrou, para êste lote, que a diferença entre as semanas restantes foi estatisticamente insignificante. $\mathrm{O}$ valor de $\mathrm{F}$, entre frangos, mostrou-se estatisticamente significante, indicando que, neste lote, houve diferença entre os frangos estudados. Esta reação individual, significativa para os frangos tratados, não pode ser atribuida, com segurança, a uma possível diferença constitucional, devido ao fato já mencionado de a experiência ter ficado algo prejudicada pelo ataque por "bouba epiteliomatosa"

\section{c) Tamanho dos testículos}

No final da experiência, todos os frangos foram sacrificados e o tamanho dos testículos analisado. A diferença entre o tamanho dos testículos dos frangos não tratados e os dos tratados foi bastante grande, de maneira a dispensar qualquer análise mais detalhada (Fig. 2). O tamanho dos testículos, em média, foi o seguinte, para os frangos não tratados: $35 \mathrm{mms}$. de comprimento $\mathrm{x} 15 \mathrm{mms}$. de diâmetro. $O$ pêso médio, de cada um, foi de $50 \mathrm{gs}$. No lote tratado, o tamanho médio foi de $15 \mathrm{mms}$. para o comprimento e $5 \mathrm{mms}$. para o diâmetro.

\section{4 - DISCUSSÃO E CONCLUSÓES}

O tratamento de aves com estrogênio é um assunto que vem recebendo, nestes últimos anos, especial atenção dos pesquisadores. Os estrogênios sintéticos, principalmente o dietilestilbestrol, mais simplesmente estilbestrol, derivado dietílíco do paradiidroxiestilbesteno, foram já experimentados tanto nas fêmeas $(6,15,16,21)$ como nos machos. O tratamento dos machos tem sido mais estudado, em vista da melhora que se obtem nas aves destinadas ao mercado consumidor de carne.

O emprêgo dêsses estrogênios pode ser feito por meio de comprimidos, implantados por baixo da pele, geralmente no pescoço das aves ou então diluido e misturado à ração. LORENZ, 1943 (8) encontrou bons resultados com comprimidos de estilbestrol implantados subcutaneamente no pescoço de frangos "Leghorn". Já o mesmo resultado, por via oral, não foi obtido por JAAP and THAYER e THAYER, JAAP and PEN- 
QUITE $(7,18,19)$ (1944). Estes autôres acharam que a potência oral do estilbestrol foi insuficiente para se obter um depósito de gordura satisfatório. Entretanto, êsses mesmos pesquisadores (7, 18 e 19) verificaram que o éter dimetílico (dimethyl ether) do estilbestrol, quando administrado, em mistura com óleo de soja, na dose de 50 miligramas por libra (454 gramas) de ração, foi uma dose ótima para a deposição de gordura nos frangos. Em 1945, LORENZ (11), experimentando o estilbestrol e o éter dimetílico de estilbestrol, oralmente, constatou que o ganho em pêso foi uniforme com ambas as drogas, mas que a implantação de comprimidos de estilbestrol foi superior à administração oral.

A nossa experiência foi feita com comprimidos de " $\mathrm{Ca}$ pastrol" (estilbestrol) lançados recentemente no mercado bras.leiro e na dose mínima de um comprimido, aplicado subcutaneamente, no pescoço de frangos "Leghorn". Nossas observaçōes permitiram concluir pelos seguintes efeitos dêsse tratamento: a) descoloração e murchamento das cristas, a partir da primeira semana de tratamento, com paralisação de seu crescimento; b) ganho médio em pêso, no final de 6 semanas de tratamento, praticamente igual para os frangos tratados e não tratados (diferença estatisticamente não significativa); c) diferença muito grande quanto ao tamanho dos testículos, que se apresentaram muito reduzidos nos frangos tratados e bem desenvolvidos nos frangos não tratados; d) melhoria na qualidade da carne, devido a uma maior deposição de gordura entre os tecidos e provável aumento de revestimento carnoso, quando comparado em relação ao desenvolvimento das ossos, que parece afetado pelo tratamento.

Nossas conclusões concordam, de uma maneira geral, com aquelas obtidas por vários outros autôres. Os frangos utilizados para a experiência tinham, inicialmente, cêrca de 3 meses de idade. $O$ efeito dos comprimidos mostrou-se nêles bastante favorável, confirmando as observações de LORENZ and BACHAMAN (13) de que o aproveitamento é melhor nas aves mais velhas (com cêrca de 12 semanas). O tratamento de frangos, de aproximadamente 3 a 4 meses oferece vantagens, do ponto de vista econômico, pois, durando o efeito de um comprimido cêrca de 6 a 8 semanas, conforme observação de BOUVIER, SARTORI e TORRES (2), torna-se desnecessária a aplicação de nova dose até o momento da ave estar em condiçöes, pela idade, de ser sacrificada.

A descoloração e paralisação do desenvolvimento das cristas são fatos observados por quase todos os demais pesquisado- 
res e serve como índice do efeito do tratamento, já na primeira semana. Assim, é possível, economicamente, aplicar nova dose, já de início, nas aves que não apresentarem essa reação, algumas vezes por perda do comprimido pelo orificio de introdução. A descoloração da crista e da face, que dá ao lote um aspecto doentio, pode ser remediada, segundo JAAP and THAYER (8) pelo emprêgo de androgênios, juntando-se à raçã excremento de vaca ou administrando-se às aves pequenas doses de methyl testosterone, nas cristas e nas faces.

No que se refere ao pêso das aves, a nossa experiência ficou àlgo prejudicada, devido ao ataque pela "bouba epiteliomatosa". Apesar disso, os resultados que puderam ser obtidos foram praticamente idênticos àqueles encontrados por outros autores. $\mathrm{Na}$ nossa experiência houve, na 2a. semana de tratamento, um ganho médio em pêso menor no lote tratado, quando comparado ao ganho do lote contrôle. Nas demais semanas, o ganho foi praticamente igual para os dois lotes e, no final da experiência, a diferença entre o ganho total dos lotes tratado e não tratado foi estatisticamente insignificante. Resultado semelhante, no que se refere ao ganho total em pêso, foi obtido por LORENZ $(9,10,12)$ e THAYER, JAAP and PENQUITE (19). Entretanto, DAVIDSON, WOLTERINK and REINEKE (3) verificaram, em perús, que o pêso final dos tratados foi menor que aquêle dos contrôles.

A influência do estilbestrol cerceando o desenvolvimento dos testículos, como aquela notada na presente experiência, foi também constatada por outros autores.

Do ponto de vista prático, os resultados mais notáveis foram aquêles constatados quanto à melhoria da qualidade da carne, em consequêhncia do tratamento. Embora essa melhoria não tenha sido, nas nosssas experiências, controlada com muito rigor, ela foi entretanto observada. Vários autores, como LORENZ $(9,10,12)$, THAYER, JAAP and PENQUITE $(18,19)$, SYKES, DAVIDSON and BARRETT (17), GLAZENER and JULL (5), DAVIS and THAYER $(4,20)$ e outros, referem-se especialmente a melhor qualidade da carne que se obtem por meio da aplicação de estrogênios, principalmente devido a um maior acúmulo de gordura que se verifica entre os diversos tecidos da ave.

A aplicação de comprimidos de Capastrol, em galos velhos, não apresentou, segundo as nossas observações preliminares, resultados muito satisfatórios, mesmo com a aplicação de dois comprimidos por cabeça. Houve uma reação apenas inicial, possivelmente devida a pequena dose aplicada. Resultados obti- 
dos por THAYER, JAAP and PENQUITE (18) mostraram algum efeito da droga, quando aplicada em galos velhos.

\section{5 - ABSTRACT}

The effect of "Capastrol", pellets of diethilstilbestrol at $0,015 \mathrm{~g}$., in 12 weeks S. C. W. Leghorn cockerels, was studied.

The results secured are in accordance with those obtained by other authors and can be resumed as follows:

1) paleness and shrunken of the comb, whose size is reduced, in comparison with the controls;

2) no differences in the total weight gain between control and treated lot;

3) size of testis greatly reduced in treated males;

4) improvement of meat by fat deposition in chickens that received treatment.

\section{6 - BIBLIOGRAFIA}

1-BIRD, S. (1949). The Mechanism of Exogenous Estrogen Activity in Male Fowl.Poultry Science 28:757.

2-BOUVIER, G., D. SARTORI e F. A. TORRES (1949). Ensaios de Castração Quimica de Frangos. $O$ Biológicó 15:31-34.

3-DAVIDSON, J. A., L. F. WOLTERINK and E. P. REINEKE (1946). Some Effects of High Dosàges of Sinthetic Estrogens in Young Turkeys.Poultry Science 25:400.

4-DAVIS, G. T. and R. H. THAYER (1948). Finishin' Mar: ket Turkeys with Estrogens. Poultry Science 27:79-83.

5-GLAZENER, E. W. and M. A. JULL (1946). Effects of Thiouracil, Desicated Thyroid and Stilbestrol Derivatives on Various Glands, Boay Weight and Dressing Appearance in the Chicken. Poultry Science 25:236-241.

6-HERRICK, E. H. (1944). Some Influence of Stilbestrol, Estrone and Testosterone Propionate on the Genital Tract of Young Female Fowls. Poultry Science 23:65-66.

7-JAAP, R. G. and R. H. THAYER (1944). Oral Administration of Estrogens in Poultry. Poultry Science 23:249-251. 
8-JAAP, R. G. and R. H. THAYER (1945). Use of Androgens to Obtain Red Comb, Face and Wattles in Estrogenfattened Chickens. Poultry Science 24:187-188.

9-LORENZ, F. W. (1943). Fattening Cockerels by Stilbestrol Administration. Poultry Science 22:190-191.

10-LORENZ, F. W. (1944). The Influence of Diethylstilbestrol on Fat Deposition in Turkeys. Poultry Science 23: 458-459.

11 - LORENZ, F. W. (1945a) The Influence of Diethylstilbestrol on Fat Deposition and Meat Quality of Chickens. Poultry Science 24:128-134.

12 - LORENZ, F. W. (1945b) The Fattening Action of Orally Administered Synthetic Estrogens as Compared with Diethylstilbestrol Pellet Implants. Poultry Science 24:91-92.

13-LORENZ, F. W. and G. H. BACHMAN (1947). Lipemia and Fat Deposition in Response to Oral Administration of Synthetic Estrogens. Poultry Science 26:419-431.

14-LORENZ, F. W. (1945). Estrogenios Para a Ceva de Aves. A Fazenda, Ano 45:58.

15-MUNRO, S. S. and I. L. KOSIN (1943). Dramatic Response of the Chik Oviduct to Estrogen. Poultry Science 22: $330-331$.

16 - PHILLIPS, R. E. (1943). Ovarian Response of Hens and Pullets to Injection of Ambinon. Poultry Science 22:368-371

17 - SYKES, J. F., J. A. DAVIDSON and F. N. BARRET (1945). The Effect of Feeding Diethylstilbestrol to Cockerels. Poultry Science 24:543-545.

18 - THAYER, R. H., R. G. JAAP and R. PENQUITE (1944). Fattening Chickens by Feeding Estrogens. Poultry Science 23:555.

19 - THAYER, R. H., R. G. JAAP and R. PENQUITE (1945). Fattening Chickens by Feeding Estrogens.Poultry Science 24: 483-495.

20 - THAYER, R. H. and G. T. DAVIS (1948). Use of Estrogens in Turkey Broiler Production. Poultry Science 27:176-181.

21 - TURNER, C. W. (1948). Feeding Estrogen (Dianisylhexene) to Laying Hens. Poultry Science 27:593-600.

22 - WAITSMAN, J. (1949). Como Fazer Capões sem Castrar Frangos. Vida Rural 3:23. 


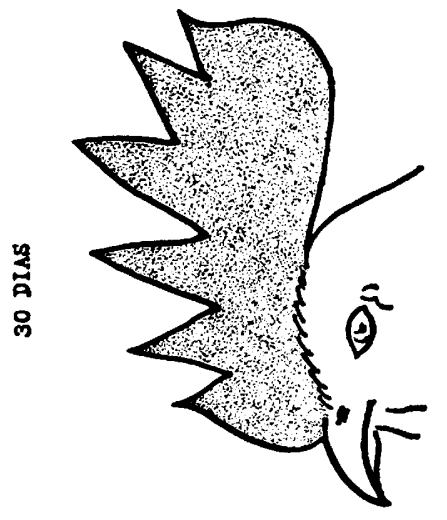

कึก

in.

"1

11

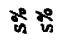

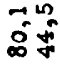

" "

$\ddot{~}$

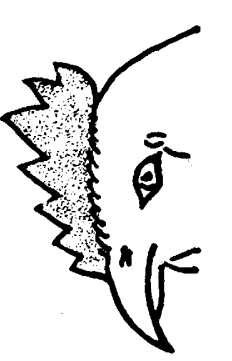

भำ

พัฒ

II

Hax

نزي

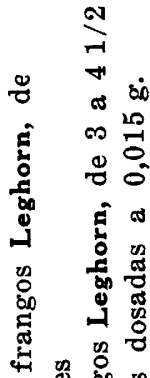

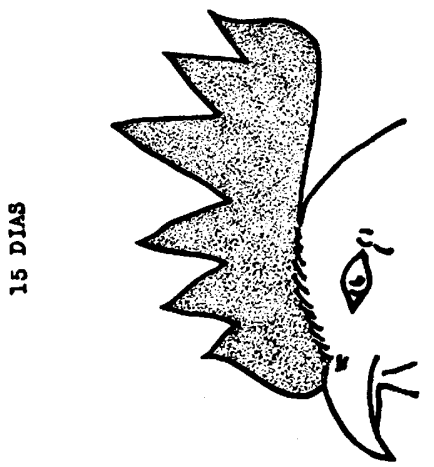

$\because 2$

ज्ञ

I"

ํํㄴ

กั

"1

$x \times$

$\ddot{0} \ddot{x}$

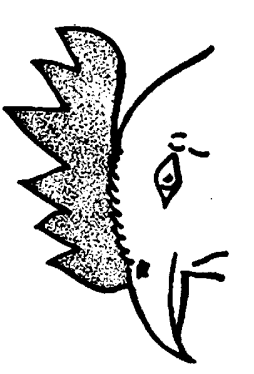

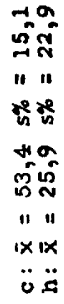

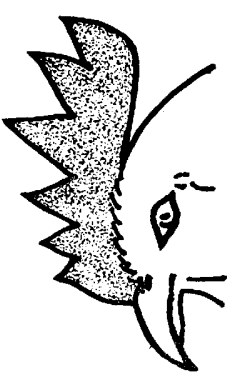

+a

¿

$+$

"1"

ํำ

क्षं

"1

$\because \ddot{a}$

옴

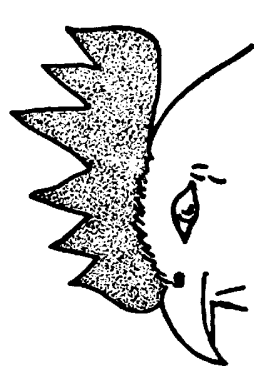

"

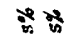

के

พิ ผ

"

IX 12

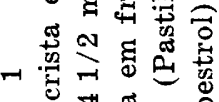

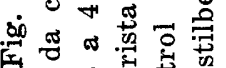

๓

壳 宓

号

\&

ดั

$\because$ के

品

ำ

$\ddot{0} \ddot{\Sigma}$ 


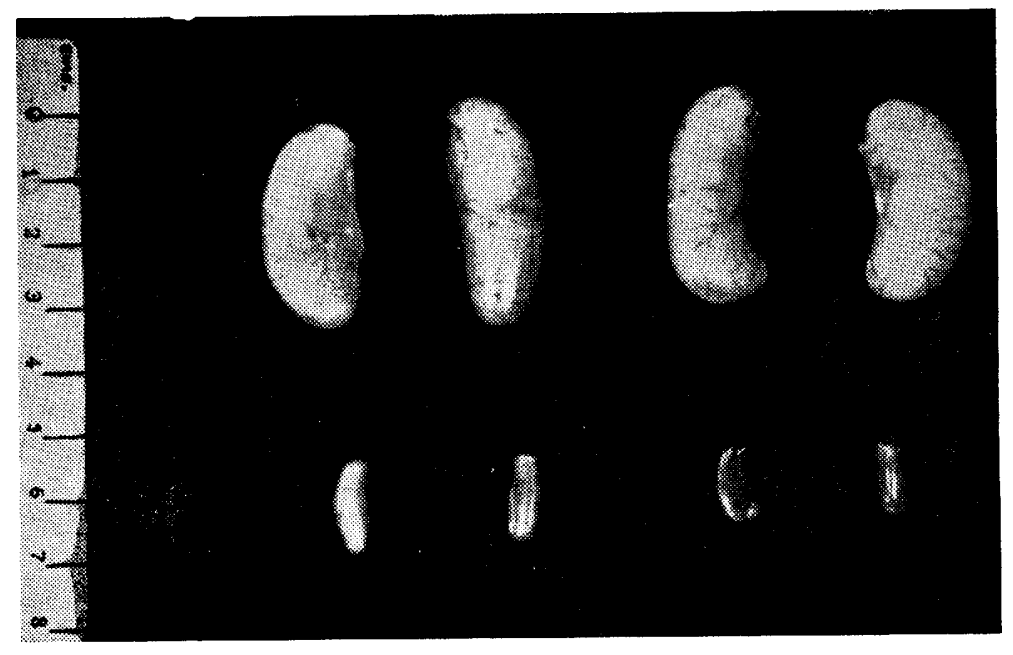

Fig. 2 - Em cima : Testiculos de dois frangos Leghorn aos $4.1 / 2$ meses de idade

Em baixo : Testiculos de dois frangos Leghorn aos 4.1/2 meses de idade, tratados com pastihas de Capastrol. 